\title{
Anthracycline Antineoplastic Antibiotic
}

National Cancer Institute

\section{Source}

National Cancer Institute. Anthracycline Antineoplastic Antibiotic. NCI Thesaurus. Code C1594.

An antineoplastic antibiotic that is structurally similar to the benzoquinone ansamycin antibiotic geldanamycin. A geldanamycin analogue binds to HSP90, a chaperone protein that aids in the assembly, maturation, and folding of proteins. Subsequently, the function of HSP90 is inhibited, leading to the degradation and depletion of client proteins such as kinases and transcription factors involved with cell cycle regulation and signal transduction. 